 \\ Cerámica y Vidrio

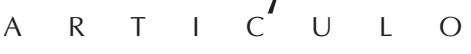

\section{Estudio de efectos dimensionales en pseudocerámicas ferroeléctricas}

\author{
M.J. MELCÓN, J. DE FRUTOS, A.M. GONZÁLEZ, C. DURO \\ E.T.S.I.Telecomunicación UPM. Ciudad Universitaria s/n. Madrid (Spain)
}

\begin{abstract}
Con el objetivo de avanzar en el estudio de la influencia de los parámetros dimensionales en las propiedades de materiales electrocerámicos, hemos fabricado materiales a los que llamaremos pseudocerámicas que, básicamente son composites de monocristales molidos embutidos en bases de diferentes polímeros. En estos materiales controlamos entre otros factores el tamaño de grano y el espesor de la matriz polimérica entre granos, disponiendo así de una amplia gama de pseudocerámicas en las que medimos y estudiamos propiedades ferro-piro-piezoeléctricas y las relacionamos con los aspectos dimensionales de las mismas. Se presentan los resultados correspondientes a la conformación del material y su respuesta eléctrica.

Palabras claves: ferro-piro-piezoelectricidad, dimensionalidad, electrocerámicas, composites

\section{Study of dimensional effects in ferroelectric pseudoceramics}

With the objective to advance in the study of dimensional properties in ferroelectric materials, we have prepared a new kind of materials, which are going to be called "pseudoceramic". These are, basically, composites that are constituted of milled single crystals packed tightly in different polymers. In these materials, we control, between other factors, grain-size and the thickness of polymeric matrix between grains. Therefore, we have a large range of pseudoceramics, over we study and measure ferro-piro-piezoelectric properties, and we related them with the dimensional aspects.
\end{abstract}

Key words: ferro-piro-piezoelectricity, dimensional properties, electroceramics, composites

\section{INTRODUCCIÓN}

Cuando hablamos de efecto dimensional en materiales cerámicos ferroeléctricos, estamos hablando de aspectos tan dispares como tamaño de grano, espesor de borde de grano, o espesor y superficie del material cerámico.

Las propiedades de los materiales cerámicos ferroeléctricos son por un lado, inherentes a la composición del material y por tanto dependen de la estructura cristalina del mismo, mientras que por otro lado están fuertemente influenciadas por la naturaleza microestructural que esta íntimamente relacionada con el procesamiento cerámico seguido $(1,2)$. Un ejemplo clásico lo constituye el valor de constante dieléctrica del $\mathrm{BaTiO}_{3}$, que presenta un máximo de 6000 para tamaños de grano de $0.7 \mu \mathrm{m}$, frente a valores inferiores a 2000 para granos con tamaño superior a $2 \mu \mathrm{m}$, con lo cual un condensador cerámico con microestructura adecuada puede duplicar el valor de capacidad almacenada para el mismo volumen de material (3).

Las estructuras de dominios estables en materiales cerámicos ferroeléctricos son resultado de un balance de energía, en particular entre la energía anisótropa, la energía de pared de dominio y las energías elásticas y eléctrica. Los primeros estudios teóricos mostraban una dependencia parabólica en la relación tamaño de dominio/ tamaño de grano. Sin embargo la distribución de tamaños de dominios presenta desviaciones para tamaños inferiores a $1 \mu \mathrm{m}$ y superiores a $10 \mu \mathrm{m}$. Considerando condiciones elásticas de borde en la relación tamaño de dominio, D, tamaño de grano, G, experimentalmente se ha observado que la relación $\mathrm{D} \sim \mathrm{G}^{\mathrm{m}}$ arroja valores de $m=1 / 3$ para los granos grandes y de $m=2 / 3$ para los granos pequeños. Sin embargo en otros sistemas ferroeléctricos como PZT, no existe esta dependencia, siendo $\mathrm{m}=1 / 3$ en todo el rango. En estos materiales, la temperatura de transición $T_{c}$ disminuye con la disminución del tamaño de grano. Sin embargo, para cerámicas ferroeléctricas laminares con estructura tipo Aurivillius, la temperatura de transición aumenta con la disminución del tamaño de grano, no encontrándose estudios sobre la estructura de dominios. (4)

Existen otra serie de aspectos en los que hasta ahora no se ha profundizado, y que representando respuestas anómalas exhiben una fuerte dependencia con el tamaño de grano. Entre ellos cabe destacar la absorción de los materiales ferroeléctricos para frecuencias en el rango de microondas, que puede dar lugar a interesantes aplicaciones como filtros. Los fenómenos que pueden justificar dicho efecto tienen un posible origen en: las vibraciones de las paredes de dominio que están controladas por el número de paredes de dominio; la resonancia piezoeléctrica del grano que esta controlada por el tamaño de grano; los modos de vibración blandos controlados por los movimientos atómicos; y la rotación de los dipolos eléctricos controlada por el anclado $(5,8)$.

No se trata sin embargo del único aspecto en que existe una fuerte controversia científica en cuanto al origen y efecto de algunas propiedades en los materiales ferroeléctricos cerámicos con el tamaño de grano. Debido al fuerte impacto tecnológico que poseen dichos efectos en las numerosas aplicaciones 
de estos materiales, estos aspectos resultan de sumo interés. $(1,3,4)$. La existencia de bordes de grano de diferente naturale$\mathrm{za}$, parece estar en el origen de la mayoría de las discrepancias encontradas. Al disminuir el tamaño de grano aumenta notablemente la influencia del borde de grano sobre las propiedades derivadas del material, debiéndose estudiar el material como un composite. Las fases que habitualmente se generan en borde de grano son de naturaleza no ferroeléctrica y suponen ruptura del flujo eléctrico. Por otro lado dichos bordes de grano representan lugar de concentración de impurezas de tipo aceptor que contribuyen al incremento de las pérdidas dieléctricas del material cerámico. $(9,11)$

La naturaleza del borde de grano ha sido profusamente estudiada en los materiales cerámicos ferroeléctricos, sin embargo existen dos lagunas importantes, la cinética de formación de fases secundarias por un lado, y la influencia de dichas fases sobre las propiedades del material por otro lado. Dado que la integración y reducción de tamaño del material requiere una fuerte disminución en el tamaño de grano, se hace necesario un estudio sistemático de las propiedades de estos materiales teniendo en cuenta el origen de las fases secundarias, así como su influencia en dichas propiedades.

Para determinar la influencia de estos factores, es necesario disponer de un amplio rango de materiales, y con un control estricto de la composición y del tamaño, tanto en el borde de grano como en su interior, pero, dada la complejidad de los sistemas cerámicos, resulta difícil disponer de sistemas absolutamente fiables.

Para avanzar en este estudio, hemos preparado un sistema que llamaremos "pseudocerámica", entre el monocristal y la cerámica. Se trata de un "composite" formado por polvo de monocristales ferroeléctricos, tamizados y en consecuencia con control del tamaño, con matrices poliméricas diferentes, en las que se varía su contenido. De esta forma, se dispone de un sistema constituido por "granos" de una composición bien definida (la del monocristal), e interfase (borde de grano), de diversos tamaños, $\mathrm{y}$ con composiciones diferentes. Presentamos resultados sobre la respuesta eléctrica de diferentes pseudocerámicas y se relacionan con los aspectos microestructurales y dimensionales de las mismas.

\section{PROCEDIMIENTO EXPERIMENTAL}

El material de partida con el que estamos trabajando, es Sulfato de Triglicina, $\left(\mathrm{NH}_{2} \mathrm{CH}_{2} \mathrm{COOH}\right)_{3} \mathrm{H}_{2} \mathrm{SO}_{4}$, conocido con el nombre abreviado de TGS. La fabricación de monocristales, se realiza por crecimiento en condiciones de disolución saturada, y temperatura controlada (de $30 \pm 0.5^{\circ} \mathrm{C}$ ))

Los monocristales así formados, seleccionados de forma que el número de maclas, defectos o inhomogeneidades sea reducido se somete a un proceso de molienda, donde se reduce a tamaños de polvo que varían desde micras hasta décimas de milímetro. Mediante un proceso de tamizado, seleccionamos el tamaño de polvo y lo clasificamos de acuerdo a la siguiente gradación:

Tamaño: mayor de $100 \mu \mathrm{m}$; entre 100 y $50 \mu \mathrm{m}$; entre 50 y 20 $\mu \mathrm{m}$, entre 20 y $10 \mu \mathrm{m}$, entre 10 y $5 \mu \mathrm{m}$ entre 5 y $2 \mu \mathrm{m}$, entre 2 y $1 \mu \mathrm{m}$, tamaño inferior a $1 \mu \mathrm{m}$.

El proceso de tamizado es muy complejo puesto que el material es muy higroscópico y tiende a apelmazarse una vez molido. Para facilitar el proceso, se introducen en todos los tamices bolas de alumina, que con el proceso de vibrado de la tamizadora, ayudan a pasar el material, e intensifican el efecto de la molienda, y dentro de la columna de tamices, se incluye bolsas de gel de sílice para que en la medida de lo posible se reduzca la cantidad de agua presente en el polvo.

El paso siguiente, consiste en conformar las muestras tratando de obtener un sistema similar a una cerámica. Los diferentes tamaños de polvo, nos permiten definir "el tamaño o tamaños de grano" conque vamos a configurar nuestro sistema, con la confianza que éste estará constituido por elementos monocristalinos, de alto grado de pureza. La fase central de la "pseudocerámica" queda así constituida.

La mayor complicación se presenta al tratar de simular el borde de grano. Con las estimaciones realizadas, el borde de grano en una cerámica, representa un volumen, que en condiciones normales y considerando la posible porosidad de una cerámica, nunca debería ser superior al $15 \%$ de la masa total. Valores mas altos nos alejarán del sistema que pretendemos estudiar, aunque en el ámbito tecnológico pueden presentar interés como "composites" de monocristales (12).

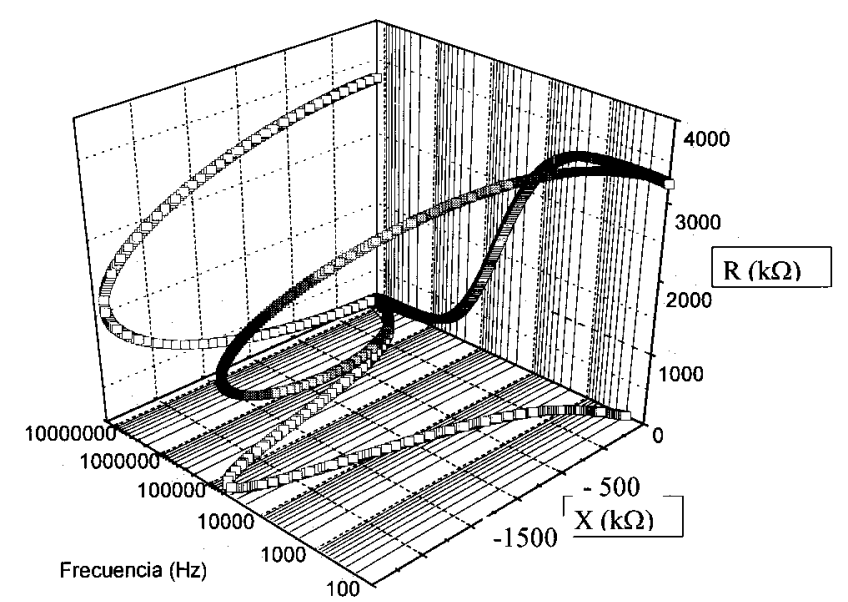

Figura 2.- Respuesta eléctrica en función de la frecuencia de una muestra no polarizada 
TABla I. PARÁMETROS CARACTERÍSTICOS DE TGS MONOCRISTALINO, SUMINISTRADOS POR EL INTERNATIONAL CENTER FOR DIFFRACTION DATA

\begin{tabular}{|c|c|c|c|c|c|c|c|c|c|c|c|}
\hline $\mathrm{d}(\mathrm{A})$ & 8.83 & 7.24 & 6.32 & 5.52 & 5.37 & 5.14 & 5.06 & 4.947 & 4.416 & 4.203 & 4.164 \\
\hline $\mathrm{hkl}$ & 100 & 110 & 020 & 101 & 001 & 120 & 111 & 011 & 200 & 201 & 121 \\
\hline Int & 20 & 10 & 14 & 20 & 4 & 20 & 40 & 50 & 25 & 50 & 12 \\
\hline$d(A)$ & 4.096 & 4.013 & 3.988 & 3.827 & 3.805 & 3.622 & 3.501 & 3.390 & 3.352 & 3.318 & 3.183 \\
\hline hkl & 021 & 101 & 211 & 111 & 130 & 220 & 221 & 121 & 131 & 031 & 040 \\
\hline Int & 45 & $<1$ & 18 & 50 & 20 & 90 & 25 & 20 & 100 & 12 & 75 \\
\hline $\mathrm{d}(\mathrm{A})$ & 3.071 & 3.051 & 2.985 & 2.977 & 2.946 & 2.908 & 2.87 & 2.863 & 2.792 & 2.761 & 2.745 \\
\hline hkl & 301 & 230 & 311 & 231 & 201 & 131 & 211 & 102 & 112 & 202 & 141 \\
\hline Int & 4 & 4 & 30 & 6 & 10 & 10 & 4 & 4 & 6 & 2 & 35 \\
\hline
\end{tabular}

Conseguir compactar muestras en estas condiciones es complicado, y para lograrlo, hemos trabajado con diferentes bases poliméricas y pegamentos (acetatos de etilo, con diferentes modificadores, resinas epóxicas de diferentes viscosidades, etc.) y cianocrilato muy fluido.

Este último, es el que nos ha proporcionado mejores resultados, puesto que permite mojar todo el material con cierta facilidad y proporciona rigidez suficiente para poder manipular y medir las muestras. El control de contenido de material, se hace añadiendo el cianocrilato en exceso para mojar todo el material y posteriormente, mediante prensado se controla la cantidad de cianocrilato eliminado de las muestras. Mediante la medida del aumento relativo en peso de la muestra, se estima la proporción de material de amalgama utilizado.

\section{RESULTADOS Y DISCUSIÓN}

La caracterización por medio de difracción de Rayos X de las muestras así conformadas, muestras un espectro de difracción característico del TGS, poniendo de manifiesto que se mantiene el carácter cristalino de las muestras.

En la Tabla I, se recogen los parámetros característicos TGS monocristalino, determinados por difracción de Rayos X y suministrados por el International Center for Diffraction Data. Para determinar si nuestro material una vez molido mantiene un comportamiento similar, se ha determinado el espectro de difracción de Rayos X para este material en el rango de 0 a 40 $2 \theta$, que corresponde a los valores de referencia de la tabla I. A partir del espectro (figura 1), determinamos los parámetros equivalentes para nuestro material. Estos datos son los que aparecen en la Tabla II

Comparando nuestros resultados con los de referencia, puede observarse un ligero desplazamiento en las posiciones de los picos, y fundamentalmente una variación en los valores de intensidad relativos a cada plano de difracción. Este resultado era de esperar, puesto que al disponer de polvo en lugar de monocristal, la proporción de planos y orientación de los mismos tiene que corresponder a una distribución uniforme sin direcciones o planos preferentes.

Una vez verificada la consistencia mecánica de las muestras, se procede a electrodarlas, y a determinar su comportamiento eléctrico en función de la frecuencia, y con diferentes campos continuos aplicados a las muestras. Las muestras caracterizadas son de polvo de TGS con un tamaño medio de 100 micras, apelmazado con un $15 \%$ de cianocrilato; tienen un espesor de $0.5 \mathrm{~mm}$ y un diámetro de $8 \mathrm{~mm}$.

Las medidas eléctricas, se han realizado con un equipo HP4291-A, con el módulo de aplicación de tensión en continua, hasta valores de $200 \mathrm{~V}$, e intensidades de hasta $2 \mathrm{~mA}$.
TABla II. PARÁMETROS CARACTERÍsticos DE TGS MONOCRISTALINO MOLIDO, DETERMINADOS EXPERIMENTALMENTE.

\begin{tabular}{|c|c|c|c|c|c|c|c|c|c|c|c|}
\hline d(A) & 16,934 & 9,418 & $\mathbf{8 , 7 9 6}$ & $\mathbf{7 , 2 2 4}$ & $\mathbf{6 , 2 9 4}$ & 5,901 & $\mathbf{5 , 5 1 7}$ & $\mathbf{5 , 3 5 4}$ & $\mathbf{5 , 1 2 5}$ & $\mathbf{5 , 0 5 0}$ & $\mathbf{4 , 9 3 7}$ \\
\hline Int & 6,00 & 9,00 & $\mathbf{1 0 8}$ & $\mathbf{6 1 , 0}$ & $\mathbf{1 2 0}$ & 15.0 & $\mathbf{1 5 8}$ & $\mathbf{3 4 , 0}$ & $\mathbf{1 5 1}$ & $\mathbf{3 2 1}$ & $\mathbf{3 2 3}$ \\
\hline d(A) & $\mathbf{4 , 4 1 3}$ & $\mathbf{4 , 1 9 4}$ & $\mathbf{4 , 0 8 5}$ & $\mathbf{3 , 9 8 3}$ & $\mathbf{3 , 8 1 8}$ & $\mathbf{3 , 6 1 8}$ & $\mathbf{3 , 4 9 7}$ & $\mathbf{3 , 3 7 9}$ & $\mathbf{3 , 3 4 7}$ & $\mathbf{3 , 3 0 6}$ & $\mathbf{3 , 1 6 1}$ \\
\hline Int & $\mathbf{1 6 3}$ & $\mathbf{3 9 8}$ & $\mathbf{3 7 6}$ & $\mathbf{1 5 5}$ & $\mathbf{3 7 7}$ & $\mathbf{7 0 5}$ & $\mathbf{2 7 5}$ & $\mathbf{2 4 1}$ & $\mathbf{9 7 4}$ & $\mathbf{1 3 8}$ & $\mathbf{8 1 1}$ \\
\hline d(A) & $\mathbf{3 , 0 4 6}$ & $\mathbf{2 , 9 7 6 8}$ & $\mathbf{2 , 9 4 3 6}$ & $\mathbf{2 , 9 0 3 1}$ & $\mathbf{2 , 8 6 3 0}$ & $\mathbf{2 , 7}$ & $\mathbf{2 , 7 4 2 9}$ & 2.6874 & 2,6271 & 2,5672 & 2,5230 \\
\hline Int & $\mathbf{5 9 , 0}$ & $\mathbf{2 9 8}$ & $\mathbf{1 0 8}$ & $\mathbf{1 3 8}$ & $\mathbf{9 1 , 0}$ & $\mathbf{8 9 5}$ & $\mathbf{4 0 2}$ & $\mathbf{2 6 9}$ & 123 & 67,0 & 61,0 \\
\hline d(A) & 2,4787 & 2,4293 & 2,4114 & 2,3447 & 2,3094 & 2,2703 & 2,2359 & 2,2022 & 2,1517 & 2,1218 & 2,0512 \\
\hline Int & 204 & 222 & 75,0 & 50,0 & 147 & 75,0 & $\mathbf{8 4 , 0}$ & $\mathbf{2 0 3}$ & $\mathbf{4 6 , 0}$ & 93,0 & $\mathbf{8 1}$ \\
\hline
\end{tabular}

La figura 2 muestra la respuesta eléctrica en función de la frecuencia de una muestra no polarizada. La Figura 3 corresponde a un estudio similar, pero con diferentes campos continuos aplicados sobre las muestras durante el proceso de caracterización eléctrica. Los datos obtenidos ponen de manifiesto un fenómeno de relajación en las muestras que es muy dependiente del campo aplicado durante el proceso de caracterización.

Hemos estudiado las propiedades eléctricas de muestras con diferente tamaño de polvo, distintas matrices y varias proporciones de polímero. La respuesta eléctrica es muy dependiente de todos los parámetros considerados. Para evitar la dispersión de resultados, y teniendo en cuenta que pretendemos muestras con una base polimérica inferior al $15 \%$ en peso, hemos establecido como parámetros fijos la composición y proporción de la matriz (cianocrilato al $15 \%$ ). En estas condiciones, hemos observado que muestras con menor tamaño de polvo, proporcionan mayores valores de permitividad dieléctrica y menores valores de pérdidas dieléctricas.

\section{CONCLUSIONES}

Hemos fabricado pseudocerámicas ferroeléctricas, de bajas pérdidas, y con control de tamaño y composición de grano (polvo de monocristales de alta pureza y con selección de tamaño mediante tamizado mecánico), y tamaño y composi-

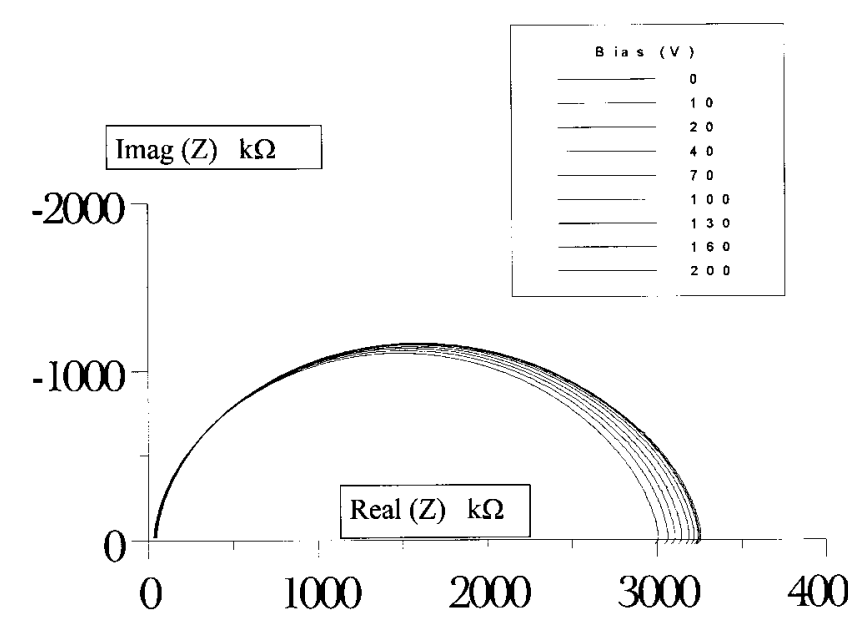

Figura 3.- Respuesta eléctrica en función de la frecuencia aplicando diferentes campos durante la medida. 
ción de borde de grano (matriz polimérica) de composición muy diferente al interior.

Hemos establecido como valor máximo de matriz polimérica el $15 \%$ del total del material. Estos niveles únicamente los hemos conseguido utilizando como base cianocrilato de alto grado de fluidez. Con esta base, hemos conformado pastillas con diferentes tamaños de "grano", que presentan rigidez mecánica suficiente para poder manipularlas, electrodarlas y caracterizarlas eléctricamente.

La caracterización eléctrica de estas muestras, pone de manifiesto que, manteniendo valores y composición de matriz similares (cianocrilato al $15 \%$ ), las muestras con menor tamaño de polvo proporcionan mayores valores de permitividad dieléctrica y menores valores de pérdidas dieléctricas.

\section{AGRADECIMIENTOS}

Este trabajo ha sido financiado a través de la CICYT por el proyecto MAT97-0694-C02.

\section{BIBLOGRAFIA}

1. M.P Wenger, P. Blanas, R.J. Shuford, D.K Das-Gupta. "Characterization and evaluation of piezoelectric composite bimorphs for in-situ acoustic emission sensors". Poly.-Eng.-and-Science. 39, 3; 508-18 (1999)

2. E.C Subbarao, "Grain size effects in advanced ceramics", Col. Sur. A: Phys. Eng. Asp. 133, 1-2, 3-11 (1998)

3. W Cao, C.A Randall. "Grain size and domain size relations in bulk ceramic ferroelectric materials", J. Phys. Chem. Sol., 57, 10, 1499-1505 (1996)

4. R. E. Newnham, G. R. Ruschan, Smart Electroceramics, J. Am. Ceram. Soc. 74(3),436-480 (1991).

5. N.Cereceda, J.A. Gonzalo, J.de Frutos, A.M. González. “Estudio de las resonancias piezoeléctricas y sus sobretonos en PZT cerámico. Aproximación microscópica". Bol. Soc. Esp. Cer. Vidrio en prensa.

6. B.D. Begg, E.R. Vance, J. Nowotny, "Effect of Particle Size on the Room Temperature Crystal Structure of Barium Titanate", J. Am. Ceram. Soc. 77(12), 3186-3192 (1994).

7. Z. Zhang, R. Raj, "Influence of Grain Size on Ferroelastic Toughening and Piezoelectric Behaviour of Lead Zirconate Titanate", J. Am. Ceram. Soc . 78(12), 3363-3368 (1995)

8. J. de Frutos, M.L. Calzada, E. Menéndez, A.M. González. “Non-uniform distribution of polarization in modified lead titanate ceramics by defects". J. Mat. Sci.: Mat Elect., 8, 327-331 (1997)

9. J.de Frutos, B.Jiménez “Distribución de polarización a partir de medidas piroeléctricas". Bol. Soc. Esp. Cer. Vidrio, 30 (5), 344-350, (1992)

10. J. de Frutos, M.L. Calzada, E. Menéndez. “A study of ionic defects in modified lead titanate ceramics". J. Mat Sci., 30, 2866-2872 (1995)

11. M.L.Calzada y J. de Frutos. "Chemical processing and microstructure of piezoelectric lead titanate ceramics". J. Mat. Sci.: Mat. Electronics. 4, pp.83-88, (1993)

12. W.A.Grandia, C.M.Fortunko, “NDE applications for Air-Coupled Ultrasonic Transducer", IEEE Ultrasonics Symposium 1, 697-709 (1995) 